\title{
A spin-polarised first principles study of short dangling bond wires on $\mathrm{Si}(001)$
}

\author{
C.F. Bird and D.R. Bowler *,1 \\ Department of Physics and Astronomy, University College London, Gower Street, \\ London WC1E 6BT, UK
}

\begin{abstract}
Short dangling bond wires (DB wires), fabricated on H-terminated Si(001) surfaces, show patterns of displacement that depend on their length. We have performed density function calculations, with and without spin-polarisation, designed to investigate the atomic and electronic structure of these wires. As expected, we find that even length wires are accurately modelled by non-spin polarised calculations, whilst odd length wires must be modelled using spin-polarised calculations. In particular, the use of spin-polarisation provides quantative agreement with STM observations, rather than the qualitative agreement reported elsewhere.
\end{abstract}

Key words: Density functional calculations, silicon

\section{Introduction}

With the continuing drive to further miniaturize microelectronics and develop realistic nanoelectronic devices, there is a great deal of interest in the atomic and electronic properties of atomic scale devices and the change in behaviour at this scale. One prototypical model for an atomic scale wire is the dangling bond wire[1], which is formed by removing a line of hydrogen atoms from one side of a dimer row on a hydrogenated $\mathrm{Si}(001)$ surface with a STM tip.

* Corresponding author

Email addresses: c.bird@ucl.ac.uk (C.F. Bird), david.bowler@ucl.ac.uk (D.R. Bowler).

URLs: http://www.cmmp.ucl.ac.uk/ cfb/ (C.F. Bird), http://www. cmmp.ucl.ac.uk/ drb/research.html (D.R. Bowler).

1 Also at: London Centre for Nanotechnology, Department of Physics and Astronomy, Gower Street, London WC1E 6BT, UK

Preprint submitted to Surface Science Letters 31 October 2018 


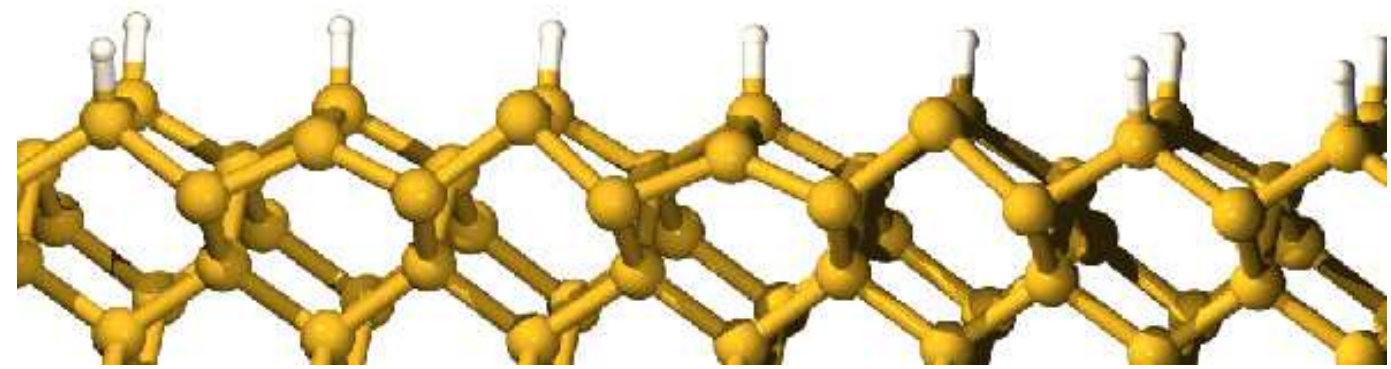

Fig. 1. A DB wire of length four, showing the DUDU pattern with one terminating hydrogen on the left and two on the right. Only the top four layers of silicon are shown.

The atomic structure of a length four DB wire is shown in Fig. 1. We present electronic structure calculations designed to investigate the atomic and electronic structure of short, finite dangling bond wires, and show that for these wires there is a marked difference between wires with odd and even numbers of atoms.

The infinite dangling bond wire undergoes a Peierls transition[2,3,4], with alternate atoms displacing up and down together with charge transferring from the down atoms to the up atoms. Finite wires should undergo a Jahn-Teller distortion[5] (since Peierls distortions only apply to infinite one-dimensional systems), and there is good STM evidence that this occurs[2], although the exact mechanism of the distortion has recently been contested[6]. Conduction in these one-dimensional systems is expected to involve polaronic effects, and it has been shown theoretically that injection of charge into the dangling bond wire results in formation of polarons[4], which are highly mobile despite their localisation[7]. We also expect soliton effects, which we have investigated elsewhere[8].

We have performed calculations within density functional theory (DFT) using spin polarisation in the generalized-gradient approximation (GGA), implemented with pseudopotentials and a plane-wave basis set. We have calculated the atomic and electronic structure of wires from two to five atoms long. It is important to note that the odd-length wires have an unpaired electron, and thus the use of spin polarisation is vitally important (and has a large effect on the results, as discussed below).

There has been previous modelling work on this system[2,4,6,9], but none of this work has considered the importance or effect of spin polarisation. Bowler and Fisher[4] and Cho \& Kleinman[6] considered flat and infinite wires with LDA and GGA respectively, and found the relative displacement of up and down atoms to be $0.67 \AA$ And 0.74 Årespectively. Watanabe et al. [9] considered infinite DB wires of different types (and their interaction with Ga), while Hitosugi et al.[2] considered the same finite wires that we model in this paper. They compared the simulation results to STM images of the wires, finding 
Table 1

Minimum total energies for different length dangling bond wires with and without spin polarisation

\begin{tabular}{l|c|c|c|c|c|c} 
Spin ? & Length 2 & Length 3 & Length 4 & Length 5 & Infinite & Hydrogenated \\
\hline No & -664.742 & -660.211 & -655.776 & -651.235 & -638.112 & -673.876 \\
Yes & -664.749 & -660.308 & -655.694 & -651.297 & -637.691 & -673.876 \\
\hline
\end{tabular}

qualitative agreement: the length $3 \mathrm{DB}$ wire shows corrugation of $\sim 0.15 \AA$ in STM, while their calculations find a corrugation of $\sim 0.50 \AA$. We will show below that agreement between simulations and observations is improved enormously by the inclusion of spin polarisation in the calculation.

The rest of the paper is organised as follows: in the next section, we present details of the computational procedure that we used; we then present the results of our modelling for the DB wires; and finally we present our conclusions.

\section{Computational Details}

We performed DFT calculations using the PW91 GGA functional[10] for exchange and correlation, both with and without spin-polarisation. We used the VASP code[11], with ultrasoft pseudopotentials, a plane wave cutoff of 200 $\mathrm{eV}$ and a $2 \times 2 \times 1$ Monkhorst-Pack[12] grid of k-points; these parameters give good energy and force convergence. The unit cell was 8 dimers long, one dimer row wide and six layers deep, with the bottom layer of atoms constrained to remain in bulk-like positions and terminated with hydrogen, giving a total of 136-144 atoms (going from an infinite wire to a totally hydrogenated surface). Calculations on even length wires (including the 8 atom cell used for the infinite wire) using spin-polarisation were constrained to a total magnetic moment of zero. While odd length wires should be only correctly be modelled with spin polarisation, we performed non-spin polarised calculations for comparison purposes (similarly, the spin polarised calculations of even length wires were run for comparison).

\section{Results}

The energetics for DB wires of lengths 2, 3, 4, 5 and an infinite wire, found both with and without spin polarisation, are presented in Table 1. This shows that the lowest energy structures (which will be analysed in detail below) for even length wires are found without spin polarisation and for odd length wires are found with spin polarisation, as might be expected. 
Table 2

Vertical displacements of atoms in dangling bond wires of different length, found using spin polarisation. Displacements are given in $\AA$ relative to hydrogenated atoms. Atoms 5-8 apply only to the atoms in the 'infinite' 8 atom cell.

\begin{tabular}{l|c|c|c|c|c|c|c|c} 
Length & Atom 1 & 2 & 3 & 4 & 5 & 6 & 7 & 8 \\
\hline 2 & -0.080 & -0.049 & - & - & - & - & - & - \\
3 & -0.016 & -0.148 & -0.016 & - & - & - & - & - \\
4 & 0.007 & -0.143 & 0.111 & -0.013 & - & - & - & - \\
5 & 0.000 & -0.132 & 0.000 & -0.132 & -0.016 & - & - & - \\
Inf & -0.058 & -0.063 & -0.083 & -0.020 & -0.113 & -0.036 & -0.068 & -0.060 \\
\hline
\end{tabular}

The displacement patterns of atoms in these wires are given in Table 2 for spin polarised calculations, and Table 3 for non-spin polarised calculations. The displacements are significantly larger without spin polarisation than with spin polarisation, and this agrees with experimental results. STM measurements of short and long wires[2] find a relative displacement from up to down atoms of $\sim 0.6 \AA$ in a 13 atom long wire, and $0.18 \AA$ in short wires. When considering STM measurements, particularly of semiconductor surfaces, one has to take into account the fact that there may well be contributions to measured heights from electronic as well as geometric effects. In the present situation, there are two factors that give us confidence that the height measured by STM linescans is the true height: first, the energies of the top-most filled states for both up and down spins, which contribute strongly to the observed features, differ in energy by only $0.01 \mathrm{eV}$ (i.e. a negligible amount); second, the images are taken at a large bias voltage $(-2 \mathrm{~V})$ where any remaining electronic effects would be largely washed out in STM line scans[13]. With the exception of the length four wire (which will be discussed below), the simulated short wire displacements in Table 2 for length three and five wires match extremely well with the measured displacements. The previous modelling of these systems[2] found displacements of $\sim 0.5 \AA$, which are a factor of four larger than the measured values, and resemble non-spin polarised calculations. We also note that our infinite wire without spin polarisation matches the long wire displacements very well.

The length four wire leaves some questions to be answered. The lowest energy is found without spin polarisation, as expected, but this has displacements from atom to atom of $0.6 \AA$, compared to experimental values of $0.1 \AA$. However, a length four wire will have two different forms, DUDU and UDUD, as well as soliton-like excitations such as UDDU (which we confirmed as existing, and being only $0.07 \mathrm{eV}$ less stable than the ground state) which suggests that the STM images are likely to be measuring an average of all these states, leading to the apparent discrepancy. A similar mechanism has been proposed before[2], though without the UDDU calculation. 
Table 3

Vertical displacements of atoms in dangling bond wires of different length, found without spin polarisation. Displacements are given in $\AA$ relative to hydrogenated atoms.

\begin{tabular}{l|c|c|c|c|c|c|c|c} 
Length & Atom 1 & 2 & 3 & 4 & 5 & 6 & 7 & 8 \\
\hline 2 & -0.057 & -0.036 & - & - & - & - & - & - \\
3 & 0.080 & -0.376 & 0.096 & - & - & - & - & - \\
4 & 0.243 & -0.441 & 0.226 & -0.344 & - & - & - & - \\
5 & 0.131 & -0.386 & 0.212 & -0.375 & 0.114 & - & - & - \\
Inf & -0.479 & 0.310 & -0.481 & 0.310 & -0.477 & 0.310 & -0.481 & 0.310 \\
\hline
\end{tabular}
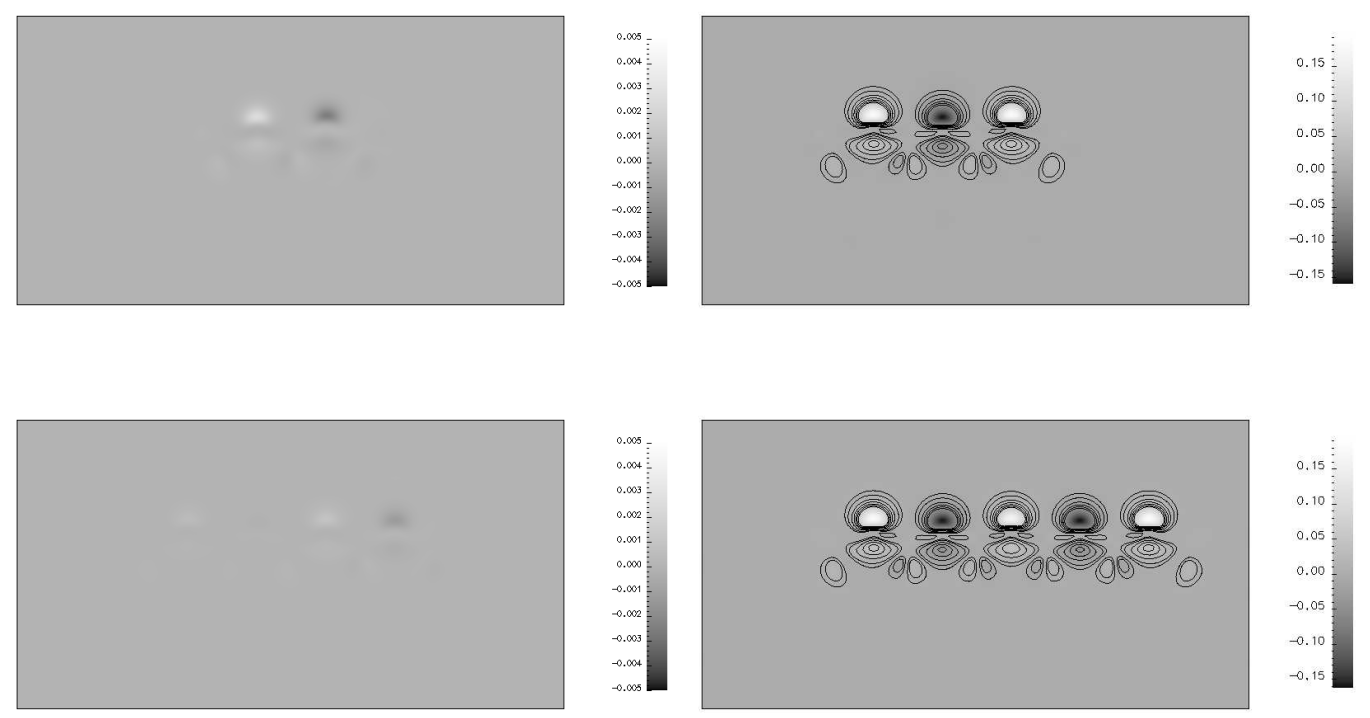

Fig. 2. Plots of spin difference density for wires of length two, three, four and five. The net spin for both length two and four is zero, while for three and five it is one. Note the different scales for odd and even length wires.

Plots of spin difference for wires of length two, three, four and five are shown in Fig. 2. As expected the results of spin-polarised calculations for odd-length wires show a strong spin Peierls effect with alternation between adjacent atoms. However there is only a negligible spin Peierls effect in even-length wire, suggesting that the energetic balance between spin difference and lattice distortion is delicate. We note that if the net spin zero constraint is removed, an up-up spin state totalling just more than 1 appears in length 2 and length 4, that are respectively just 7 and $90 \mathrm{meV}$ more stable. 


\section{Conclusions}

In this paper we have presented the results of DFT-GGA simulations of finite length dangling bond wires on the $\mathrm{Si}(001)-(2 \mathrm{x} 1)$ surface. We have found that for odd length wires, a spin polarised state with relatively small displacements $(\sim 0.13 \AA)$ of the atoms is most stable. These displacements are in good agreement with those reported from STM observations[2] that are in the order of $0.18 \AA$. These results contrast with both our own non-spin polarised simulations and those reported previously[2] that give displacements in the order of $0.5 \AA$.

In contrast, for even length (and infinite periodic) wires, a non-spin polarised state is the most stable. The relative displacement of wire atoms increases strongly as a function of wire length, from $0.02 \AA$ for length 2 to $\sim 0.6 \AA$ for length 4 to $\sim 0.8 \AA$ for an infinite wire. This is in good qualitative agreement with STM observations[2] which range from $0.18 \AA$ for short wires to $\sim 0.6 \AA$ in a 13 atom long wire. However, the difference between experimental and simulated displacements is marked for length 4 wires.

We speculate that this disparity between STM measurements and simulations results from averaging of a number of states in STM measurements. As well as the perfectly ordered UDUD and DUDU wires, we have shown[8] that solition-containing states are situated at energies very close to the ground state. Dynamical processes may result in a mixture of these being measured by the STM process. Further dynamic simulation of the system and of the interaction between the surface and STM tip may clarify the situation.

We conclude that accurate modelling of finite length dangling-bond wires requires the use of spin-polarised methods for odd length systems and non-spin polarised approaches are suitable for even length systems. We speculate that, given appropriate conditions, spin-polarisation in such systems might be observable by spin-polarised STM[14].

\section{References}

[1] T.-C. Shen, C. Wang, G. Abeln, J.R.Tucker, J. Lyding, P. Avouris, R. Walkup, Science 253 (1995) 1590.

[2] T. Hitosugi, S. Heike, T. Onogi, T. Hashizume, S. Watanabe, Z. Q. Li, K. Ohno, Y. Kawazoe, T. Hasegawa, K. Kitazawa, Phys. Rev. Lett. 82 (20) (1999) 40344037.

[3] R. Peierls, Quantum Theory of Solids, Clarendon Press, Oxford, 1955, p. 110. 
[4] D.R.Bowler, A.J.Fisher, Phys. Rev. B (2001) 035310.

[5] H.A.Jahn, E.Teller, Proc. Roy. Soc. A 161 (1937) 220.

[6] J.-H. Cho, L.Kleinman, Phys. Rev. B 66 (2002) 235405.

[7] M. Todorovic, A. Fisher, D. Bowler, J. Phys: Cond. Mat. 14 (2002) L749.

[8] C.F.Bird, A.J.Fisher, D.R.Bowler, Submitted to Phys. Rev. B Condmat/0301594.

[9] S.Watanabe, Y.A.Ono, T.Hashizume, Y.Wada, Surf. Sci. 386 (1997) 340-342.

[10] J. Perdew, J. A. Chevary, S. H. Vosko, K. A. Jackson, M. R. Pederson, C. Fiolhais, Phys. Rev. B 46 (1992) 6671.

[11] G.Kresse, J.Furthmüller, Comp. Mat. Sci. 6 (1996) 15.

[12] H. Monkhorst, J. Pack, Phys. Rev. B 13 (1976) 5188.

[13] W.A.Hofer, A.J.Fisher, G.P.Lopinski, R.A.Wolkow, Phys. Rev. B 63 (2001) 085314 .

[14] M. Bode, M. Getzlaff, R. Wiesendanger, Phys. Rev. Lett. 81 (19) (1988) 4256. 